\title{
Author Correction: Hepatorenal syndrome
}

Pere Ginès, Elsa Solà, Paolo Angeli, Florence Wong, Mitra K. Nadim and Patrick S. Kamath

Nature Reviews Disease Primers 4, Article number: 23 (2018) https://doi.org/10.1038/s41572018-0022-7 Published online 13 September 2018

The original version of this article omitted an initial from the name of contributing author Patrick S. Kamath, who was listed as Patrick Kamath. The article has now been corrected.

https://doi.org/10.1038/s41572-018-0035-2। Published online: 15 October 2018 